\title{
Endoscopic Closure After Endoscopic Resection for Superficial Non- Ampullary Duodenal Tumors
}

\author{
Satoshi Tanabe ${ }^{1}$ and Takuya Wada ${ }^{2}$ \\ ${ }^{1}$ Department of Advanced Medicine Research and Development Center for New Medical Frontiers, Kitasato University School of \\ Medicine, ${ }^{2}$ Department of Gastroenterology, Kitasato University School of Medicine, Kanagawa, Japan
}

See "The Use of Endoscopic Clipping in Preventing Delayed Complications after Endoscopic Resection for Superficial NonAmpullary Duodenal Tumors” by Jee Young An, Byung-Wook Kim, Joon Sung Kim, et al., on page 563-569.

Superficial non-ampullary duodenal epithelial tumors (SNADETs) are less common than most other gastrointestinal tumors, ${ }^{1}$ but with the widespread use of routine endoscopic examination, they are now more frequently encountered in daily clinical practice. ${ }^{2}$ However, several aspects related to SNADETs have not yet been fully understood, including their level of clinical malignancy and natural history, endoscopic diagnosis, and the indications and methods for endoscopic therapy.

Similar to other gastrointestinal tumors, the indications for endoscopic resection of SNADETs are highly atypical adenoma and intramucosal carcinoma without lymph node metastasis. Duodenal tumors are rare, and although advances have been made in endoscopic diagnostics such as image-enhanced endoscopy, the accuracy of qualitative diagnosis and invasion depth diagnosis remains unsatisfactory. Furthermore, the accuracy of the pathological diagnosis differs depending on the pathologist. While there have been problems such as discrepancies between preoperative biopsy diagnoses and histological

Received: April 8, 2021 Revised: April 19, 2021

Accepted: April 19, 2021

Correspondence: Satoshi Tanabe

Department of Advanced Medicine Research and Development Center for New Medical Frontiers, Kitasato University School of Medicine, 1-15-1, Kitasato, Minamiku, Sagamihara city, Kanagawa 252-0374, Japan

Tel: +81-42-778-8111, Fax: +81-42-778-8390, E-mail: s-tanabe@kitasato-u.ac.jp ORCID: https://orcid.org/0000-0002-9207-5637

(c) This is an Open Access article distributed under the terms of the Creative Commons Attribution Non-Commercial License (http://creativecommons.org/ licenses/by-nc/3.0) which permits unrestricted non-commercial use, distribution, and reproduction in any medium, provided the original work is properly cited. diagnoses after endoscopic therapy, reports on endoscopic resection of SNADETs have increased recently. ${ }^{3}$

Handling the endoscope in the duodenum is more difficult than in other areas of the gastrointestinal tract because of its anatomical characteristics, and the risk of adverse events, such as postoperative bleeding and delayed perforation, is reported to be high. ${ }^{4}$ Anatomically, the duodenum has a narrow lumen, and it is influenced by respiratory and gastric movements, resulting in poor scope handling and difficulty in securing a visual field. In addition, the duodenal wall is thin, and the presence of Brunner's glands leads to poor elevation following local injections (e.g. hyaluronic acid etc), increasing the risk of intraoperative perforation. Furthermore, delayed bleeding and perforation can occur because of exposure to pancreatic juice or bile. To minimize these adverse events, endoscopic therapy should be selected based on lesion size. According to Yamasaki et al., ${ }^{5}$ local recurrence can be minimized by performing endoscopic mucosal resection (EMR) or cold snare polypectomy for lesions <10 mm in size, underwater EMR for those 10-20 $\mathrm{mm}$ in size, and endoscopic submucosal dissection for those $\geq 20 \mathrm{~mm}$ in size.

Various methods have been used to prevent complications. Endoscopic clipping is the most widely used method. ${ }^{6}$ An et $\mathrm{al}^{7}$ compared a clip group with a non-clip group, and found fewer delayed adverse events in the former and recommended clipping as a useful method. The usefulness of clipping depends on the level of completeness of suturing with clips. Complete suturing can prevent adverse events, but incomplete suturing does not. Complete clipping is possible for muscosal defects up to a certain size; it may be difficult to suture defects 
completely using clips alone if it exceeds $3 \mathrm{~cm}$ in size. Attempts have also been made to combine sutures with devices such as detachable snares. ${ }^{8}$ Recently, the over-the-scope clip system (OTSC System; Ovesco Endoscopy AG, Tübingen, Germany) has also been used. ${ }^{9}$ This system can reliably suture large mucosal defects due to its strong gripping force, but the high unit price may be an issue. Furthermore, although this is still at the research level, a method of directly suturing the mucosal defect using a needle and thread has also been attempted. ${ }^{10}$ Although the report is based on performance in an animal stomach, this method is more reliable than suturing with clips; however, the technique takes time and its use in areas with a narrow lumen and poor operability, such as the duodenum, is thought to be extremely difficult.

Endoscopic resection of SNADETs is less invasive than open surgery, but carries a higher risk of serious adverse events than endoscopic resection of tumors in the stomach or esophagus. With the growing aging population, the need for endoscopic therapies is expected to increase even further. There is a need for the continuous development of reliable and simple suturing techniques for mucosal defects.

Conflicts of Interest

The authors have no potential conflicts of interest.

Funding

None.

Author Contributions

Writing-original draft: Satoshi Tanabe

Writing-review \& editing: Takuya Wada
ORCID

Satoshi Tanabe:

https://orcid.org/0000-0002-9207-5637

Takuya Wada:

https://orcid.org/0000-0001-5500-4242

\section{REFERENCES}

1. Jepsen JM, Persson M, Jakobsen NO, et al. Prospective study of prevalence and endoscopic and histopathologic characteristics of duodenal polyps in patients submitted to upper endoscopy. Scand J Gastroenterol 1994;29:483-487.

2. Goda K, Kikuchi D, Yamamoto Y, et al. Endoscopic diagnosis of superficial non-ampullary duodenal epithelial tumors in Japan: multicenter case series. Dig Endosc 2014;26 Suppl 2:23-29.

3. Kakushima N, Ono H, Takao T, Kanemoto H, Sasaki K. Method and timing of resection of superficial non-ampullary duodenal epithelial tumors. Dig Endosc 2014;26 Suppl 2:35-40.

4. Honda T, Yamamoto H, Osawa H, et al. Endoscopic submucosal dissection for superficial duodenal neoplasms. Dig Endosc 2009;21:270-274.

5. Yamasaki Y, Uedo N, Takeuchi Y, Ishihara R, Okada H, Iishi H. Current status of endoscopic resection for superficial nonampullary duodenal epithelial tumors. Digestion 2018;97:45-51.

6. Hoteya S, Furuhata T, Takahito T, et al. Endoscopic submucosal dissection and endoscopic mucosal resection for non-ampullary superficial duodenal tumor. Digestion 2017;95:36-42.

7. An JY, Kim BW, Kim JS, Park JM, Kim TH, Lee JS. The use of endoscopic clipping in preventing delayed complications after endoscopic resection for superficial nonampullary duodenal tumors. Clin Endosc 2021;54:563-569.

8. Lee BI, Kim BW, Kim HK, et al. Routine mucosal closure with a detachable snare and clips after endoscopic submucosal dissection for gastric epithelial neoplasms: a randomized controlled trial. Gut Liver 2011;5:454-459.

9. Mori H, Ayaki M, Kobara H, et al. Suitable closure for post-duodenal endoscopic resection taking medical costs into consideration. World J Gastroenterol 2015;21:5281-5286.

10. Goto O, Sasaki M, Ishii H, et al. A new endoscopic closure method for gastric mucosal defects: feasibility of endoscopic hand suturing in an ex vivo porcine model (with video). Endosc Int Open 2014;2:E111-E116. 